\title{
Chylous ascites after lymphadenectomy in a Wilms' tumor patient
}

\author{
Mustafa Asım Yörük ${ }^{1}$, Meryem Erat-Nergiz², Çetin Timur ${ }^{1}$, Aylin Canbolat-Ayhan ${ }^{1}$, \\ Müferet Ergüven ${ }^{1}$ \\ Department of ${ }^{1}$ Pediatric Oncology and Hematology, Medeniyet University Göztepe Training and Research Hospital, Istanbul; \\ ${ }^{2}$ Department of Pediatrics, Yildırm Beyazıt University Yenimahalle Training and Research Hospital, Ankara, Turkey. \\ E-mail: meryemerat@hotmail.com \\ Received: 11th May 2017, Revised: 28th July 2017, Accepted: 17th September 2017
}

SUMMARY: Yörük MA, Erat-Nergiz M, Timur Ç, Canbolat-Ayhan A, Ergüven M. Chylous ascites after lymphadenectomy in a Wilms' tumor patient. Turk J Pediatr 2018; 60: 436-438.

Wilms' tumor is the most common renal malignancy in children and the fourth most common childhood cancer. It accounts $6-7 \%$ of all childhood malignancies. Surgical resection is an important therapy option and transabdominal or transperitoneal resection with lymph node sampling is preferred.

Development of chylous ascites following intraabdominal or retroperitoneal resection in pediatric age group generally results from extensive lymph node dissection, accidental ligation or interruption of lymphatic ducts. Diseases or conditions affecting abdominal and/or retroperitoneal lymph nodes may cause chylous ascites. Postoperative chylous ascites is associated with significant morbidity and may cause mechanic, nutritional and immunological complications.

In the present study, a 16-month-old infant with Stage IV Wilms' tumor who developed chylous ascites after left nephrectomy and lymphadenectomy will be presented; chylous ascites treatment with enteral nutrition and surgical treatment approach for Wilms' tumor will be reviewed.

Key words: chylous ascites, Wilms' tumor, lymphadenectomy.

Chylous ascites is the accumulation of milky chyle in the peritoneal cavity. Ascitic fluid triglyceride level greater than $110 \mathrm{mg} / \mathrm{dl}$ is diagnostic of chylous ascites. ${ }^{1-3}$ Chylous ascites is a rare postoperative complication in pediatric surgery. Development of chylous ascites following intraabdominal or retroperitoneal resection in the pediatric age group generally results from extensive lymph node dissection, accidental ligation or interruption of lymphatic ducts. In the present study, a 16-monthold infant with Stage IV Wilms' tumor who developed chylous ascites after left nephrectomy and lymphadenectomy will be presented; chylous ascites treatment with enteral nutrition and surgical treatment approach for Wilms' tumor will be reviewed.

\section{Case Report}

A 14-month-old male was admitted to a local hospital due to hematuria and abdominal mass. Abdominal ultrasonography and abdominal computerized tomography revealed a mass of the left kidney. Chest X-ray and computerized tomography were normal. Left radical nephrectomy and ureterectomy was performed. Unfortunately, intraoperative tumor spillage occurred during the operation. Wilms' tumor with anaplasia was diagnosed on histopathological examination. The patient was offered chemotherapy and radiotherapy but the patient's family wanted to continue treatment at another center. Two months after diagnosis, he was admitted to our clinic with abdominal distension. Abdominal ultrasonography and computerized tomography revealed a $14 \mathrm{~cm}$

Presented at the XVII. National Pediatric Oncology Congress, 01.05.2012, Abant, Bolu, Turkey. 
mass and chest computerized tomography showed pulmonary metastases. The patient was operated again. The mass was removed totally with microscopic involvement of the resection margins and lymph nodes were dissected. Pathology of the recurrent resected mass revealed triphasic Wilms' tumor with omental and pancreatic invasion. He was treated with vincristine, actinomycin-D, adriamycin, etoposide and radiotherapy according to the Turkish Society of Pediatric Oncology protocol. He did not receive preoperative chemotherapy. Radiotherapy was performed (whole abdomen 10.5 Gy and flank 10.5 Gy) following surgery. Sudden onset of abdominal distention occurred forty days after surgery. The patient's abdomen was distended and he had respiratory distress. Abdominal circumference was $53 \mathrm{~cm}$ and weight was $12.5 \mathrm{~kg}$. The patient's weight was $8.5 \mathrm{~kg}$ a month ago. Abdominal ultrasonography and abdominal computerized tomography revealed massive ascites particularly in the pelvic region. Abdominal paracentesis yielded a $200 \mathrm{ml}$ milky ascitic fluid with high triglyceride $744 \mathrm{mg} /$ $\mathrm{dl}$, protein $4.8 \mathrm{~g} / \mathrm{dl}$, albumin $3.07 \mathrm{~g} / \mathrm{dl}$, LDH 226 U/L. Simultaneous laboratory results showed serum total protein was $6.9 \mathrm{~g} / \mathrm{dl}$, albumin was $3.9 \mathrm{~g} / \mathrm{dl}$ and LDH was $319 \mathrm{U} / \mathrm{L}$. Radiotherapy was interrupted due to ascites. Oral spironolactone was administered and the infant was initially fed with $1 \mathrm{~g} / \mathrm{kg}$ lipid containing total parenteral nutrition (TPN) and $1 \times 10 \mathrm{ml}$ oral MCT oil as breast feeding was continued. On the 5th day of TPN, the ascites increased, therefore TPN and breast feeding were stopped and $100 \mathrm{kcal} / \mathrm{kg} /$ day of "energy mixture" formula containing $10 \%$ protein, $30 \%$ MCT oil, 1-3\% PUFA (Polyunsaturated fatty acids), $60 \%$ carbohydrates was started. Energy mixture contained the following: $6 \mathrm{ml} \mathrm{MCT}$ oil, 2 scoops of protein powder (Protifar $\left.{ }^{\circledR}\right), 5$ scoops of carbohydrate powder (Fantomalt ${ }^{\circledR}$ ) mixed with water. The total volume was 170 $\mathrm{ml}$ and $1 \mathrm{ml}$ of walnut oil was added to the mixture twice a day with the interval of 12 hours. Laboratory tests including vitamins A, D, $\mathrm{E}, \mathrm{K}$, prothrombin time and INR were repeated and found in normal range. On the 3rd day of the treatment, abdominal circumference was 48 centimeters and on the $15^{\text {th }}$ day $38 \mathrm{~cm}$, weight was $8.2 \mathrm{~kg}$. Abdominal ultrasonography revealed no ascites. Egg, fat-free vegetable puree, fat-free milk, chicken and fish were restarted gradually. On the 30th day MCT oil and formula were stopped and radiotherapy continued. No abdominal distension or ascites were developed again. He was in remission for 4 years and he did not come for followup visits later. Written informed consent was obtained from patient's mother for this case presentation.

\section{Discussion}

Wilms' tumor is the most common renal malignancy in children. Surgical resection is an important part of the therapy. Lymph node sampling is sufficient and extensive lymph node dissection is not required because cysterna chili can be damaged during nephrectomy.

Chylous ascites is an uncommon surgical complication characterized by accumulation of lymphatic fluid in peritoneal cavity and it can result from congenital lymphatic defects, inflammatory processes, neoplasms, surgical procedures or trauma. Chylous ascites is very infrequent accounting for between $1 / 20000$ - 1/100000 of all hospital admissions. ${ }^{1,2}$ Ascitic fluid triglyceride level greater than $110 \mathrm{mg} / \mathrm{dl}$ is diagnostic of chylous ascites. ${ }^{3}$ Development of chylous ascites following retroperitoneal surgery is a rare complication causing significant morbidity and occurs due to extensive lymph node dissection, accidental ligation or interruption of lymphatic ducts. Postoperative chylous ascites represents a difficult management problem due to mechanical, nutritional and immunological consequences of the constant loss of protein and lymphocytes. ${ }^{4}$ Abdominal aorta surgery is the most common cause of chylous complications and occurs following retroperitoneal lymphadenectomy and nephrectomy in testis and kidney cancer patients. 4,5 Postoperative chylous ascites has been infrequently described after nephrectomy for Wilms' tumor.6,7 Extensive lymph node dissection, particularly above the level of the renal hilum, appears to be associated with the development of postoperative chylous ascites. ${ }^{7}$

According to the National Wilms' Tumor Study guidelines, lymph node sampling should be performed rather than retroperitoneal lymphadenectomy in patients with Wilms' tumor. ${ }^{7}$

The interval between surgery and development of chylous ascites varies from a few days to several months.1,4 Our patient underwent an extensive lymph node dissection and chylous ascites developed 4 weeks after the surgery due to impaired lymphatic drainage. 
The management of the patients with chylous ascites is controversial, since it is a rare condition and there are different effective treatment options including both noninvasive (e.g. diet modifications) and invasive (e.g. radiographic interventions, surgery) procedures. In a previous study, $67 \%$ of 156 patients were treated with conservative methods and $33 \%$ of them required surgical intervention. ${ }^{8}$ Therapeutic paracentesis and diet modifications are the conservative treatment methods. Protein and MCT rich diet modification improves lymphatic leakage in $50 \%$ of the patients. ${ }^{4}$ The diet can be continued after the resolution of the ascites ${ }^{4,5}$ and 2 to 6 weeks TPN can be applied in refractory patients. ${ }^{5,9}$ Some authors suggest that somatostatine and/or its analogues (octreotide) can be used solely or combined with TPN in patients with chylous ascites. ${ }^{10-12}$ Invasive methods are indicated when conservative treatment methods are not useful. Lymphoscintigraphy or lymphangiography provide anatomical information, identify the leakage and allow image-guided sclerotherapy or surgical exploration to be performed. Matsufiji et al. ${ }^{13}$ reported a patient with recurrent chylous ascites who was treated with peritoneovenous shunt following failure of therapy with MCT oil and TPN.

Both TPN and surgery have some disadvantages. TPN is a potential risk factor for infections and inadequate calorie intake. In our patient breast feeding were continued along with MCT and TPN to maintain adequate calorie intake, however, it interfered with the therapy due to high fat concentrations. Although surgery is an effective treatment method, it should be considered as the last step because of the invasiveness.

Treatment with MCT containing diet is noninvasive and does not increase the risk of infection, particularly in immunocompromised patients; therefore, it appears to be the most reasonable treatment option. Chylous ascites can be treated giving the patient a medium-chain triglyceride rich diet, providing adequate calorie. MCTs do not contribute to the formation of chylomicrons and do not flow through the lymphatics, thus they do not cause chylous ascites formation. Moreover, one should be careful regarding a deficiency of fat-soluble vitamins in chylous ascites.

In conclusion chylous ascites can be diagnosed easily but effective treatment requires a long period. An algorithmic treatment protocol should be followed; starting with diet modification with MCT and subsequent TPN. The "energy mixture" formula containing $10 \%$ protein, $30 \%$ MCT oil, 1-3\% PUFA (Polyunsaturated fatty acids), $60 \%$ carbohydrates is an effective formula for infants. Lymphangiography or surgery must be performed for refractory cases.

Lymph node sampling should be performed rather than retroperitoneal lymphadenectomy in patients with Wilms' tumor.

\section{REFERENCES}

1. Evans JG, Spiess PE, Kamat AM, et al. Chylous ascites after post-chemotherapy retroperitoneal lymph node dissection: Review of the M. D. Anderson experience. J Urol 2006; 176(4 Pt 1): 1463-1467.

2. Garcia-Correa G, Aguilar-Sibilla CA, Murguia-Corral R, Espinosa-de los Monteros P. Conservative management of postoperative lymphatic fistula. Cir Cir 2005; 73: 307-310.

3. Talluri SK, Nuthakki H, Tadakamalla A, Talluri J, Besur S. Chylous ascites. N Am J Med Sci 2011; 3: 438-440.

4. Liebovitch I, Mor Y, Golomb J, Ramon J. The diagnosis and management of postoperative chylous ascites. J Urol 2002; 167 (2 Pt 1): 449-457.

5. Baniel J, Foster RS, Rowland RG, Bihrle R, Donohue JP. Management of chylous ascites after retroperitoneal lymph node dissection for testicular cancer. J Urol 1993; 150(5 Pt 1): 1422-1424.

6. Eberl W, Kwee T, Mau G. Chylous ascites after tumor nephrectomy with retroperitoneal lymphadenectomy. Monatsschr Kinderheilkd 1991; 139: 47-50.

7. Weiser AC, Lindgren BW, Ritchey ML, Franco I. Chylous ascites following surgical treatment for Wilms' tumor. J Urol 2003; 170(4 Pt 2): 1667-1669.

8. Aalami OO, Allen DB, Organ $\mathrm{CH}$ Jr. Chylous ascites: a collective review. Surgery 2000; 128: 761-778.

9. Combe J, Buniet JM, Douge C, Bernard Y, Camelot G. Chylothorax and chylous ascites following surgery of an inflammatory aortic aneurysm. Case report with review of the literature. J Mal Vasc 1992; 17: 151-156.

10. Bhatia C, Pratap U, Slavik Z. Octreotide therapy: A new horizon in treatment of iatrogenic chyloperitoneum. Arch Dis Child 2001; 85: 234-235.

11. Leibovitch I, Mor Y, Golomb J, Ramon J. Chylous ascites after radical nephrectomy and inferior vena cava thrombectomy. Successful conservative management with somatostatin analogue. Eur Urol 2002; 41: 220222.

12. Huang Q, Jiang ZW, Jiang J, Li N, Li JS. Chylous ascites: Treated with total parenteral nutrition and somatostatin. World J Gastroenterol 2004; 10: 25882591.

13. Matsufuji H, Nishio T, Hosoya R. Successful treatment for intractable chylous ascites in a child using a peritoneovenous shunt. Pediatr Surg Int 2006; 22: 471-473. 